Rabaska

Revue d'ethnologie de l'Amérique française

\title{
Fringues et frusques. Texte d'Yvette Aquin, Fille de la sagesse
}

\section{Yvette Aquin et Jean Simard}

Volume 3, 2005

URI : https://id.erudit.org/iderudit/201712ar

DOI : https://doi.org/10.7202/201712ar

Aller au sommaire du numéro

Éditeur(s)

Société québécoise d'ethnologie

ISSN

1703-7433 (imprimé)

1916-7350 (numérique)

Découvrir la revue

Citer ce document

Aquin, Y. \& Simard, J. (2005). Fringues et frusques. Texte d'Yvette Aquin, Fille de la sagesse. Rabaska, 3, 83-101. https://doi.org/10.7202/201712ar d'utilisation que vous pouvez consulter en ligne.

https://apropos.erudit.org/fr/usagers/politique-dutilisation/ 


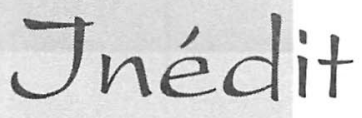

\section{Fringues et frusques \\ Texte d'Yvette Aquin, Fille de la sagesse}

\section{PréSENTÉ PAR JEAN SimARD}

Société québécoise d'ethnologie

Le patrimoine religieux recouvre plusieurs champs et il en est un qui fait l'objet d'attention plus que tous les autres de la part des ethnologues, c'est celui du patrimoine immatériel. La mémoire des savoirs et des savoir-faire religieux est bien sûr menacée du fait que le personnel vieillit et qu'il n'y a plus de relève. Cette perte se fait sentir plus cruellement dans les communautés religieuses de femmes, plus portées que les hommes à conserver et à transmettre les paroles et les gestes hérités des fondatrices. "Grâce à la structure communautaire de la vie consacrée, selon la Commission pontificale pour les biens culturels de l'Église, les religieux présentent un témoignage significatif, et toujours renouvelé, des charismes particuliers des fondateurs », et «lorsqu'une communauté religieuse vit intensément son charisme, celui-ci s'irradie également dans les formes visibles de la culture et de l'art et celles-ci semblent contaminées quelque peu par l'intensité spirituelle de ces témoins ${ }^{1} \gg$. On ne pourrait mieux dire sur les liens qui unissent patrimoines matériel et immatériel, ni sur le fait que le patrimoine immatériel donne sens au matériel. Le texte ci-dessous traduit précisément cette irradiation de l'esprit dans les formes visibles de la culture et de l'art et c'est la raison principale qui me motive à le publier. Il fait l'éloquente démonstration qu'un costume religieux, en l'occurrence celui des Filles de la sagesse, ne serait rien qu'un lot d'étoffes et d'épingles si l'une des religieuses qui en a fait usage pendant une quarantaine d'années n'avait pris la peine de le décrire dans sa symbolique autant que dans sa fonctionnalité et sa matérialité.

«Fringues et frusques² » a été écrit en 1978 par Yvette Aquin, appelée sœur Rose du Saint-Sacrement dans la communauté des Filles de la sagesse

1. Commission pontificale pour les biens culturels de l'Église, Lettre aux révérendes mères générales et aux révérends pères généraux, Rome, 10 avril 1994.

2. Je dois la communication et la libre disposition du texte et de la photographie de sœur Yvette Aquin à son neveu François Rousseau que je remercie. 


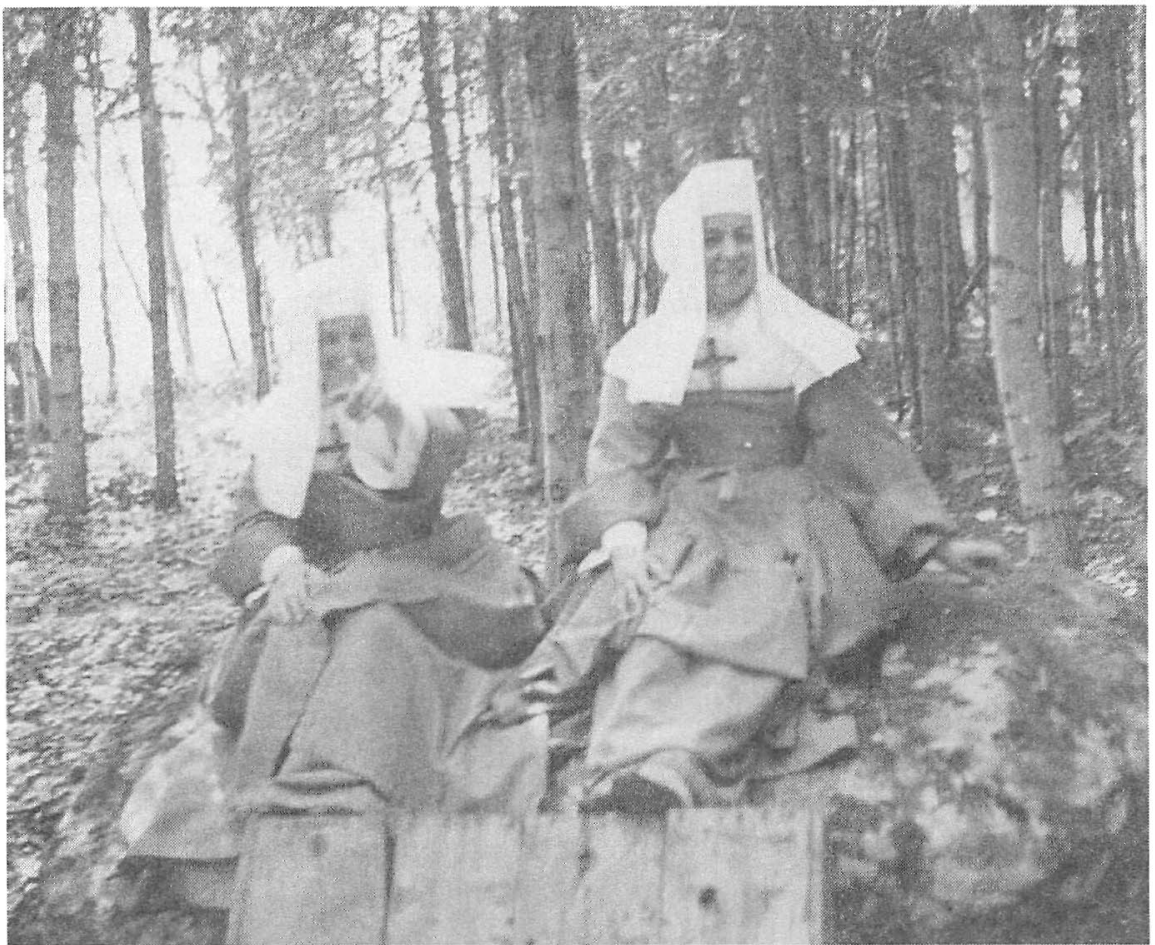

Sœur Yvette Aquin (à droite) revêtue du costume des Filles de la sagesse dans sa belle trentaine. "Mon "saint habit", écrit-elle, venait bel et bien exorciser mon élégance de toute sa séduction d'antan et de saper les fondements de mon "sexappeal "irrémédiablement effondré !!! »"

jusqu'au concile Vatican II. Elle nait dans le quartier Saint-Henri à Montréal le 23 décembre 1907 et décède le 10 août 1999 à Nicolet, où elle est inhumée. Issue d'un milieu modeste - son père Théodore Aquin était commis-vendeur chez Morgan à Montréal -, elle ambitionne d'être infirmière. En 1929, elle entre en communauté et exerce la profession d'infirmière pendant 27 ans : à Mont-Joli tout d'abord, ensuite à Papineauville, à Ottawa, à Ozone Park (New York) et à Sturgeon-Falls. Ayant une santé plutôt fragile, elle délaisse les soins aux malades vers l'âge de 50 ans et s'adonne par la suite à l'étude de la spiritualité (Université de Montréal, 1960) et de la littérature contemporaine (Campus Notre-Dame-de-Foy à Cap-Rouge, 1962), puis donne du service dans les bibliothèques de l'hôpital Sainte-Justine de Montréal et d'une autre maison de sa communauté située à Dorval. Sœur Yvette s'intéresse donc aux livres et à la littérature. D'après la notice nécrologique que sa communauté lui a consacrée ${ }^{3}$, elle était une « lectrice assidue des propos littéraires et

3. Archives des Filles de la sagesse, Ottawa. Je remercie sœur Françoise Laporte de m'avoir transmis cette notice avec plusieurs autres documents à caractère biographique. 
méditatrice de l'Évangile et des Psaumes ». Cette remarque aide encore à comprendre l'attention qu'elle porte dans son texte à des auteurs aussi étrangers aux habitudes de lecture des sœurs qu'Henrik Ibsen et Jean-Paul Sartre, qu'elle cite parmi d'autres. On le verra, sœur Yvette est une femme cultivée et elle a l'humour incisif. Elle s'est cultivée semble-t-il après sa vie active d'infirmière et a pu de la sorte prendre de la distance par rapport à sa formation première reçue au noviciat. En somme, comme tant d'autres religieux et religieuses des années de la Révolution tranquille, elle se sera développée après avoir remisé son costume des Filles de la sagesse. Quand elle rédige " Fringues et frusques », Yvette Aquin a 71 ans et peut maintenant écrire : « je me surpris à jauger jusqu'où pouvait atteindre un certain ostracisme clérical aux œillères bornées, aux partis-pris tels qu'on se croyait mordicus détentrices du record de l'intelligence et de la... perfection ! » Alors elle était véritablement devenue fille de la sagesse.

Les Filles de la sagesse sont fondées en France en 1703 et s'implantent au Canada en $1884^{4}$. Elles s'occupent d'éducation, d'hospitalisation et d'œuvres de charité. Branche féminine des pères Montfortains, dont elles partagent la spiritualité et la direction du supérieur général, elles ont leur siège principal à Saint-Laurent-sur-Sèvre, en Vendée. Sœur Yvette y a fait d'ailleurs un séjour en 1935. Le centre de la province du Canada et des ÉtatsUnis est à Ottawa. C'est dans cette ville que sœur Yvette fait son noviciat sous la gouverne de la mère française Marie de l'Assomption, qui y fut la première maîtresse des novices de 1904 à 1938. Les noviciats hors les murs de l'Hexagone seront dirigés selon le principe de « l'Union à la Congrégation " par des maîtresses françaises choisies parmi les plus fidèles à la tradition. Ce qui fait dire à sœur Yvette que les us et coutumes de 1703, " ç'avait toujours force de loi envers et contre tout aggiornamento ». Et ainsi du costume.

4. Guy Laperrière, Les Congrégations religieuses. De la France au Québec, 1880-1914, Tome 3 : Vers des eaux plus calmes, 1905-1914, Québec, les Presses de l'Unversité Laval, 2005. 


\title{
Fringues et frusques
}

\author{
À nos sœurs qui ne se prennent pas trop au sérieux... \\ "L'humour a quelque chose de libérateur... » (Freud) \\ YveTte AquiN \\ f.d.l.s.
}

À l'instar des jeunes nobles du Moyen Âge qui (recevant de leur parrain un coup sur la nuque) devenaient chevaliers, munis ultérieurement d'armure et d'équipement, de même, toute novice, chez nous, recevait - non pas un coup sur l'occiput - mais plutôt un poids de vingt-cinq livres de fringues réparties, au p'tit bonheur, sur toutes ses surfaces planes, courbes, réglées, développables... cependant qu'étourdie sous le choc, la pauvre ! tentait de remonter à la surface-tout-court de son anatomie littéralement ensevelie sous d'anachroniques vêtements.

Investiture mémorable que celle de la prise de possession de notre fief d'étamine, de bure, de laine, de flanelle, de molleton, toile, coton, coutil, fanons de baleine, lacets, cordonnets, galons, chamois, cuir et... vingtsix épingles ! Ah ! mes aïeux ! pour un « adoubement » c'en était un, et de classe !

Notre costume religieux n'était pas ce qu'on appelle un « trois-pièces " mais plus précisément un... « quatorze-pièces ».

Dans l'ensemble, une solide «pièce-montée » aux reliefs architecturaux accusés et sans bavures.

Pour ma famille, une "pièce-de-musée » digne de figurer auprès des cariatides et des atlantes de la ville de Péloponnèse.

$\mathrm{Si}$, au début du noviciat, faute d'accoutumance, on se mouvait « toutd'une-pièce », c'est néanmoins « en-pièces-détachées » que j'étale le vêtement en son entier.

- Un gilet en flanelle écrue nous couvrait les épaules jusqu'à la taille, les manches très larges s'arrêtaient aux coudes.

-Une chemise en toile toujours écrue, plissée au cou et tombant jusqu'aux chevilles, avait toute l'élégance d'une longue... poche. Par souci de précision, je signale dès maintenant la largeur de toutes nos manches, c.-à.-d. : seize pouces ; et leur longueur : trente pouces ! vous avez bien noté : trente pouces! 
(à l'exception des manches du gilet de flanelle plutôt courtes). Vous vous demandez pourquoi ces longueurs ?... moi aussi, figurez-vous !!! On se contentait de rabattre ces verges en deux plis sur les avant-bras.

Entre parenthèses, cette fameuse chemise, on la portait nuit-et-jour-dusamedi-au-samedi-suivant. Mais pourquoi ? demandez-vous encore ?... Les « us » ma chère ! Les « us et coutumes » datant de 1703, ç'avait toujours force de loi envers et contre tout « aggiornamento »... À cette lointaine époque, l'hygiène corporelle semblait fort suspecte... elle s'apparentait à la recherche de ses aises, à l'immortification, au sensualisme, patati, patata ! Bref ! les soins du corps rentraient dans le domaine de l'interdit, sujet tabou par excellence. Or, donc, vive la fidélité à l'absence totale de la plus élémentaire hygiène ! et sus à la propreté ! Douteriez-vous de mes dires ?... je vous sers, à l'instant, un flash-back historique : deux bains en vingt et un mois et, en vitesse et, revêtue d'une... chemise !! Par contre, jamais de lavage des cheveux durant toute cette longue période ! La toilette habituelle consistait à se laver à l'eau froide devant un minuscule lavabo, chacun étant muni d'une misérable débarbouillette et d'une... pierre-ponce ! dans quelle tenue ?... quelle question! Tout naturellement revêtue de nos deux chemises (la courte et la longue) de nos trois jupes (celle de coton, celle de molleton et celle de bure) tandis qu'une large pointe de cou (genre poncho) nous recouvrait le torse depuis les épaules jusqu'à la taille, par surcroît de modestie, paraît-il ! et la cérémonie se déroulait chaque soir, en famille, devant toutes les novices : les six ou sept qui se lavaient devant leur cuvette et les autres qui attendaient à la queue leu leu derrière nous, car les lavabos étaient tous alignés dans un corridor attenant au dortoir. Cette grande tolérance de ce lavochage accordé à la " nature » était de surcroît chronométrée par une maîtresse de noviciat. L'excès en tout étant un défaut avait marmonné un vieux proverbe... Mais au fait, pourquoi avoir exigé plus de chichi dans le protocole de nos libations quotidiennes ?... À la guerre comme à la guerre ! «Les preux ne dorment-ils pas couchés dans leur armure ?» (V. Hugo).

- Venait la maîtresse-pièce de notre habit religieux : le corset ! On eut pu l'appeler gond, charnière, pivot, point du front où s'articulaient les éléments du système stratégique du costume (c.-à-d. : galons, poches, brassière, tablier, pièce), mais c'était avant tout et surtout le plus abracadrabrant des salmigondis que l'on puisse boutiquer !... et, pour les mordus de l'artisanat « made in France », un échantillon du plus beau cru de la ville de Poitiers !...

Figurez-vous une pièce conçue en guise de soutien-gorge à armature de fanons de fer et de fanons de baleine juxtaposés à la douzaine les uns à côté des autres... Une de ces charpentes solides, d'une rigidité inflexible, un vrai mur de soutènement pour notre remblai... pectoral ! Entre initiées, nous 
appelions ça, nos « p'tites volailles »... parce que, une fois étalées, ces carcasses ressemblaient étrangement aux poitrines de ces... bestioles !

J'ai comme une vague idée que nos corsetières de l'an de grâce 1703 devaient descendre en ligne directe de la Monarchie de Juillet née sur les... barricades, car nos « devantures » en étaient ni plus ni moins que de parfaites répliques miniatures. Sans doute, étions-nous considérées comme des... pucelles à protéger (!) tandis que, dans le camp opposé, s'ameutaient sans vergogne les « ligueurs, frondeurs, insurgés » de tous acabits, tous les gros " méchants » quoi ! On quittait le monde parce que le monde était " méchant $» . .$. ne fallait-il pas dresser contre lui murailles, clôtures, rangées de pieux, semblables à celles que les colons du Nouveau Monde dressaient contre les Iroquois ?.. Vous vous souvenez des palissades dont nous parlait not' p'tite Histoire du Canada ?... c'était pas des charmilles, hein ?... ben nos corsets non plus ! pis c'était pas le moment de s'inviter en minaudant : ("Allons sous la charmille où l'églantier fleurit. »V. Hugo). Hélas, pas question de charmes, encore moins de parfum ou de rose $!$ du corset, émanaient plus souvent des effluves capiteux de la race ovine et pour cause ! Nos " p'tites-volailles-barricades-palissades ", pour être moins intolérables et blesser le moins possible, étaient obligatoirement capitonnées à l'intérieur par... devinez ? (je vous le donne en mille !) de la bourre de laine grossière, non cardée, jaunâtre et suintante à souhait... ensuite, par du papier journal ; des rames de vingt mains, en vouliez-vous ?... il y en avait des monceaux de découpés et, de toutes les éditions ! J'ai, en effet, lư un en-tête daté de 1860 , tenez-vous bien ! il s'agissait de déchirures du « Figaro »... qu'il se soit agi du journal satirique, politique et littéraire ou du « Figaro de Beaumarchais », je n'ai pu le vérifier, étant trop occupée à calmer ma rate qui se dilatait, se dilatait dangeureusement... car le « grand silence » qui devait toujours régner dans l'atelier de la corsetterie, rendait impossible toute communication entre les novices. Il me fallait continuer à penser « in petto » au barbier malicieux, rusé, railleur qui devait pester de dépit au fond de ce réduit insolite...

Sur d'autres déchirures-découpures de journaux, mes compagnes ont pu lire « ...ure de France " 1810 ; nous sûmes plus tard qu'il s'agissait du «Mercure de France ». Quelques autres ont déchiffré : «L'Humanité », « Le Petit Var », « Le Petit Provençal », «Le Progrès du Nord ». Il y avait également, et en abondance, des pages et des pages du « Nouveau Larousse Universel Illustré » !... L'ahurissement me gagnait ! Eh quoi ! pensais-je, l'« Encyclopédie-Dictionnaire-Universel $»$, avili au point de servir de bourre avec le " Canard Sauvage », "L'Ennemi du Peuple » du grand norvégien Henrik Ibsen !!! Par quel itinéraire labyrinthique, ou à la suite de quelle malencontreuse coalition d'archivistes et de bibliothécaires en mal de grève, toutes 
ces œuvres vinrent-elles à échouer dans le busc rigide d'une petite bretonne ignare, innocente et simplette ?... Le sabordage et le vandalisme existaientils déjà aussi florissants à l'époque qu'ils le sont dans l'actuel Québec de 1978 ?... Quelle précocité ! quand on croit toujours avoir tout inventé soimême !

À partir de cette réflexion, ma pensée dériva et je me surpris à jauger jusqu'où pouvait atteindre un certain ostracisme clérical aux œillères bornées, aux partis-pris tels qu'on se croyait mordicus détentrices du record de l'intelligence et de la... perfection. ! Insensiblement, on glissait vers une dureté qui s'enorgueillissait de « mépriser » l'amitié des êtres du monde... Ne nous enseignait-on pas, en effet, sans le moindre discernement, que c'était plaire à Dieu que de " mépriser-le-monde » (que faisait-on de ces mots de Jean : « Dieu a tant aimé le monde qu'il lui a donné son Fils ? »...) et qu'une bonne religieuse - entre autres choses saugrenues - devait exclure toute conversation ou lecture " politique et frivole " (les deux sujets sur le même palier). Dès lors, devenait compréhensible le sort réservé aux paperasses journalistiques et littéraires classées, ou plutôt coincées à jamais dans nos « devantures » sans jamais espérer de mise... à l'étalage !...

« Mais cheminons sans trop d'écart dans l'axe de notre sujet » (Barrès). Justement ! j'y arrive à l'axe de la question. Au beau milieu du corset était fichée une tige de bois (un demi-pouce par douze pouces de long). C'était comme qui dirait " l'étrave »... le pieu saillant dressé, formant proue en maintenant le devant du corset. Pour éviter le trop grand inconfort de cet ajout à la raideur hiératique, on en entortillait surtout l'extrémité inférieure (toujours avec les mêmes ingrédients : guenilles, laine, bure), puis, on baptisait du nom de « poire » cette proéminence élargie, arrondie, drue et ferme, à la mine saugrenue au possible ! At last, to give the finishing touch, aux « barricades-palissades-p'tites-volailles », on recouvrait tout le bastringue d'une toile à matelas écrue, laquelle toile disparaissait à son tour sous de larges empiècements de bure ou de peaux de... chamois !!! Ben oui ! v'la-ty-pas qu'à côté des moutons d'Australie... des baleines du Grand Nord... s'amenaient les chamois des Pyrénées !... Un peu plus, c'était le safari et la concurrence au... cirque de Moscou !!!

Pour terminer la laborieuse description de cette confection de " grandcouturier ", je signale que, flanqué au creux du corset - toujours pour adoucir le contact rugueux du bois -, s'ajustaient deux patentes en tissu de coton archi-épais, archi-raide, superposé trois ou quatre fois sur lui-même, oh ! disons, ayant la forme d'une quelconque figure polygone à trois côtés : isocèle ?... scalène ?... rectangle ?... Si vous êtes fort en géométrie, débrouillezvous ! nous, nous appelions ça, « la Poche du d'vant »... c'était cousu à la 
bourre avec du gros fil $n^{\circ} 10$, en deux pans, je dirais deux vannes d'écluses munies d'œillets métalliques. L'usage de cette manivelle ?... tantôt nous étouffer... tantôt nous libérer la taille selon qu'on laçait ou délaçait les vannes.

Lorsque notre « étau-corset » nous happait depuis les épaules jusqu'à la taille, commençait la difficile manœuvre du laçage dorsal des interminables galons dans un chassé-croisé d'œillets, lesquels nous ficelaient solidement depuis 4 heures du matin jusqu'à 9 heures du soir notre vie durant... Bardées, blindées, cuirassées, l'échine raidie, la taille moulée comme une gaine, tout... galbe anéanti à jamais ! nous atteignons enfin ! au gabarit traditionnel rêvé par les fondateurs de notre Institut. Bien que les « chères sœurs » du noviciat n'aient jamais cessé de nous inculquer fortement l'abc de la vertu extérieure de modestie (tête légèrement inclinée, yeux baissés, mains dans les manches) hélas ! dès que nous nous mettions en marche, deux à deux, pour une quelconque cérémonie, nous ressemblions à s'y méprendre à une levée de fiers boucliers courant sus aux hordes ennemies... Mais passons à une autre pelure...

- Le jupon écru en invariable tissu de coton, large et plissé (dix-huit plis) à la taille et tombant jusqu'aux chevilles.

- La jupe écrue de molleton pelucheux (!), même nombre de plis, même largeur et même longueur.

- La jupe grise : la plus grosse, la plus lourde pièce d'artillerie de notre matériel vestimentaire : cinq verges d'épaisse et rugueuse bure serrées à la taille (dix-huit plis énormes !) et retenues par une monumentale agrafe de cuivre jaune ou rouge. Cette jupe - soit dit en passant nous durait la vie entière! sa longueur s'arrêtait à un pouce du sol.

- La brassière : genre de justaucorps très ancien, toujours en épaisse bure grise ajustée à la taille " au-plus-que-possible », court en avant et muni en arrière de basques fendues en deux pans plissés (largeur quatorze pouces ; longueur seize pouces) « not' coat-à-queue » disait-on. Les manches hautes et bouffantes aux quatre plis sur les épaulettes mesuraient seize pouces de largeur et... trente pouces de longueur ! ce qui sous-entend qu'on ne s'est jamais senties gênées aux entournures. En dehors des heures de travail, où les interminables manches étaient relevées sur les avant-bras en deux larges plis (ce qui donnait quatre rangées d'épaisseur de coton, quatre rangées d'épaisseur de bure sur les bras), on baissait lesdites manches pour la prière, pour se présenter à l'Autorité, pour demander ses permissions à genoux, pour recevoir une réprimande, enfin, faire sa coulpe et baiser la terre... Combien de fois le «baissez-vos-manches ! 》 a retenti à nos oreilles de novices distraites, tel un refrain usé aux tonalités mornes, au cours de notre entraînement aux us et coutumes ancestrales !... Pas étonnant que nos 
vêtements aient été en partie couverts de « barbes » (mot-jargon signifiant : longs reprisages) aux pliures et plissures au nombre impressionnant ! Ma foi ! on passait son temps à plier, déplier, replier les huit plis à la coiffe ; les quatre plis à chaque épaule ; les huit sur les avant-bras ; les dix-huit plis de chaque jupe, en tout cinquante-quatre ; enfin les dix-huit du tablier; en touttotal : quatre-vingt-huit !

Le géologue le moins prévenu aurait identifié du premier coup d'oeil : « plissements-soulèvements-hercyniens-géologiques-du-primaire-del'époque-carbonifère ! »...

Quoi qu'il en soit, les plis - sans oublier la lourdeur, la chaleur et l'épaisseur écrasante de toutes ces pelures - semblent avoir été une des caractéristiques de notre costume religieux médiéval. Mais il y a plus ! À part les vrais plis, il y avait également tous les faux-plis que subissent des étoffes qui ne connaissent jamais le repassage. En effet, il fut deux siècles pendant lesquels on n'utilisa jamais le fer à repasser sinon pour la coiffe, et encore ! cette pauvre coiffe aperçue sur de vieilles lithographies du temps, fait piètre mine et sa forme est plutôt... moche ! Tout le reste de nos vêtements était - après lavage - étendu sur des surfaces planes, humecté, tapé, étiré « à-la-main » et à « tours-de-bras », enfin, plié de manière à ce que ça soit le plus... potable ! Ce qui explique que nous étions plus ou moins dégrossies ; le fignolage, ça n'a jamais été la coquetterie entretenue au grand jour chez les Filles de la Sagesse. Nous étions plutôt du genre... tonnelets ambulants.

- La pièce : plastron pectoral rectangulaire amovible (dix pouces par sept pouces) en bure doublée de coton et maintenue sur la poitrine à l'aide de six épingles d'acier d'un pouce et quart de long.

Porter la pièce a constitué un véritable adoucissement quant au poids du costume. Cette licence introduite tardivement dans nos coutumes, à savoir : ne plus être astreintes à porter en tout temps le lourd tablier de cérémonie, nous fut accordée pas tellement pour nous soulager de son fardeau respectable, mais tout simplement pour en retarder l'usure et ménager ce fort beau tissu de laine importé de France, comme d'ailleurs tout le reste du fourniment.

-Les poches de sœurs : pour cet item j'ai réservé quatre pages humoristiques à leur sujet, lesquelles pages accompagnent « Fringues et Frusques » en fin d'article.

- Le tablier : représentez-vous une belle ampleur aux cinq verges de largeur plissées à la taille (dix-huit plis) et recouvrant presqu'entièrement l'énorme jupe de bure (dotée du même nombre de verges et de plis) sans oublier le jupon de dessous et le molleton pelucheux aux dix-huit plis chacun ; le tout plissé autour d'une seule et unique taille de jeune fille normalement constituée. Autant vous le dire tout de go, ladite jeune fille, émergeant de ces monticules superposés, apparaissait soudain comme affligée 
d'éléphantiasis des pays chauds... ! Et c'était plutôt démoralisant pour le papa et la maman de la jouvencelle, laquelle jusqu'à hier encore, était passable !... Les chères maîtresses du noviciat avaient beau assurer les auteurs de nos jours que, désormais, " toute la beauté de la fille du Roi est audedans... » ces derniers, peu convaincus, s'évertuaient à retrouver un dernier petit reste d'air de famille à leur progéniture.

- J'ai dit un mot de la pièce, plus haut, laquelle nous a dispensées du port habituel du tablier, mais lorsque nous le portions, il fallait, aux heures de travail - et cela sous peine d'accroc à la sainte pauvreté - retrousser cette lourdeur en la fixant à l'aide d'épingles, à mi-chemin, entre la... rotule et le petit trochanter ! et revêtir cet invraisemblable amoncellement d'étoffes par un second... tablier en gros coton écru non repassé !!! C'est dire que l'ère des crinolines a longtemps perduré dans notre Institut. Était-ce en souvenir de l'impératrice Eugénie ou de nos délicieuses arrière-mères-grands ?... Quoi qu'il en soit, si on était pauvre, on n'a jamais manqué d'étoffe ni... d'envergure ! Pour m'amuser, j'ai vraiment tenté d'évaluer, par approximations successives, ma circonférence, pas la curviligne de ma surface mais bien l'épaisseur de ma... périphérie et ce, en piquant mes longues épingles d'acier d'un pouce et quart à l'horizontale, à partir de l'extérieur, en maints endroits... jamais je n'ai réussi à atteindre l'épiderme de mes hanches! Il n'y a pas à dire, si l'habit ne fait pas le moine, il semble bien le protéger ! Mais à quoi bon râler comme je le fais ! Sartre n'a-t-il pas écrit " qu'il est de règle aux U.S.A que les beaux quartiers glissent du centre à la... périphérie. " Sans le savoir, nous étions « up-to-date » depuis au-delà de deux bons siècles. Hourra! Olé !...

- Les souliers : c'est beaucoup dire ! savates conviennent mieux. Je dirai que nous étions chaussées de « sabots » de cuir noir, à talon bas découvert, sans le moindre soutien pour l'arche du pied. Ces chaussures fabriquées « à la main » par les frères-religieux-de-notre-Institut pesaient chacune une livre et ça prenait vingt à trente ans pour les mettre hors d'état de servir !

- Les bas étaient tricotés à la main avec de la laine la plus rugueuse que j'aie encore touchée... Neufs, ces bas étaient entièrement gris. À mesure qu'ils s'usaient, on ne prêtait plus la moindre attention à la teinte initiale. On tricotait avec tous les bouts de laine utilisables rencontrés.

Survenait ici une autre invention issue du plus lointain des âges... Il va sans dire que " l'Ordre de la Jarretière » n'avait pas cours en Bretagne, cependant que son insigne, à la Sagesse, semblait dater de presque la même époque. Au lieu donc de faire comme tout le monde du $\mathrm{XX}^{\mathrm{e}}$ siècle, c.-à-d. porter des petites bandes élastiques qui maintiennent et entourent les bas audessus des genoux, nous, nous tricotions d'interminables longueurs de laine grise (un pouce par trois quarts de verge.) On roulait quatre à cinq fois cette 
pseudo-jarretelle au-dessous du genou, on rabattait le bout de bas sur cet entortillement, laissant toujours le genou à nu (?)... et hop ! vous aviez le plus beau galbe de jambes « stratifiées » horizontalement de blanc, de grisbleu, de gris-fer, de gris-délavé et de blanc-beige... mais c'était en pure perte, nos chères bonnes jupes nous traînant sur les talons voilaient ces particularités saugrenues.

- Le mouchoir de cou : ce que d'ailleurs on appelle la guimpe. C'était une belle pièce de pure toile blanche épaisse, d'une verge carrée que l'on pliait deux fois en triangle, qu'on ajustait à l'aide de plis au cou, de plis aux épaules, de manière à recouvrir tout le torse avant-derrière.

- La coiffe : composée d'un bonnet en gros coton doublé ; d'une cornette, tissu en toile rectangulaire pliée en quatre sur le crâne et retombant de chaque côté des joues ; de la coiffe proprement dite en toile transparente toujours pliée en deux, le tout disposé en rectangle tout autour du front, des tempes et des joues.

Un vieux patient taquin m'avait dit un jour : «Vous seriez une ben belle p'tite sœur si vous aviez pas c'te magnére de corridor autour de la figure ! »... Ce corridor rectangulaire mesurait trente-huit pouces de longueur et une fois replié, six à sept pouces de profondeur, autant de largeur. Au lieu de dire : plisser sa coiffe, on disait : " casser » sa coiffe. On y comptait dix « cassures » et treize épingles qui maintenaient le fragile échafaudage dont les extrémités nous battaient les épaules comme des ailes de... pingouins ! $\mathrm{Ah}$ ! si vous aviez soupçonné ce que représentait " se coiffer " pour toute fille-de-la-Sagesse et la " flascure » (empesage de centaines et centaines de cornettes et de coiffes) ! Une de ces corvées compliquées et harassantes et méticuleuses qu'on n'oublie pas sa vie durant !...

- La cape : véritable houppelande en étamine noire plissée (neuf plis) à l'arrière de la tête. Sans manches, d'une ampleur majestueuse, toujours dans les cinq verges de largeur et plissée dans le dos et sur les épaules de quatorze à seize larges plis. Cette cape nous enveloppait corps et membres, en balayant royalement le sol derrière nous telle une traîne imposante. Oh ! ce n'est pas que nos maîtresses de noviciat ne nous aient pas enseigné le petit geste rituel et discret par lequel nous devions la relever avec aisance... mais, comme le dit Pierre Loti, «La cape était déjà dure à tenir... » par-dessus tout le costume si lourd ! Aussi, n'ai-je jamais pu rire... sous cape, je me contentais, résignée, de suer à grosses gouttes, tellement j'étouffais, ainsi encapuchonnée. Et puis, dites-moi, comment oser «se divertir sous cape » (Balzac) quand votre vénérable Coutumier vous invite instamment à considérer cet austère vêtement noir comme « un suaire qui doit vous ensevelir aux yeux du monde » (sic) ?... 
Après l'endossement intégral des " quatorze-pièces » du costume religieux, $\mathrm{j}$ 'aurais bien voulu me voir de pied en cape... n'ayant jamais encore eu l'occasion de me mirer - tout miroir étant prohibé pour protéger la fragilité de nos modesties embryonnaires -. Avant donc de descendre pour la cérémonie, je ne pus résister au prurit de la tentation d'un regard oblique dans le « carreau » d'une fenêtre... Imprudence catastrophique !... L'espace d'une seconde, ma grâce d'état vacillait... j'avais la frousse de ma vie... Une de ces terreurs paniques qui menacent de mettre en fuite tous vos régiments de promesses de fidélités pour « le-meilleur-et-pour-le-pire »!... C'est que je m'apparaissais soudain comme un char d'assaut... une véritable enceinte fortifiée !... contre qui ?... je vous le demande ?... C'est qu'on ne peut tout savoir le matin de sa prise d'habit, vous en convenez, n'est-ce pas ?... et, pour délurée que je fus, je n'étais pas tellement « calée » sur tous les trucs inédits des bandes sarrasines embusquées - paraît-il - derrière les murailles du couvent pour nous tomber dessus, dès que nous en franchirions l'escarpe !

En cette minute historique, où je contemplais, ahurie, l'ampleur de mes avatars, j'assistais, impuissante, au sauve-qui-peut de mes grâces juvéniles... Bien qu'extérieurement j'aie été couverte de pelures, intérieurement, je me sentais évidée comme une tige de sureau, et dans l'impossibilité d'émettre même un gémissement... Mon « saint habit » venait de bel et bien exorciser mon élégance de toute sa séduction d'antan et de saper les fondements de mon « sex-appeal » irrémédiablement effondré !!!

Cette minute de stupeur écoulée, mes vingt ans qui avaient assisté, stoïques, au sabordage de toutes mes flottes de féminité... réussirent à galvaniser leur énergie restante pour se cramponner comme à une bouée insubmersible, à la boutade de Rostand : «Moi, c'est moralement que j'aurai mes élégances. " Puis, je fonçai dans la lice, tel un paladin en quête de prouesses et d'actions généreuses, bien décidée à livrer des « barouds d'honneur » en joutant de courage et d'endurance sous mon " caparaçon » moyenâgeux, lourd, inconfortable, et calorifuge s'il en fut !... Et, sans plus tergiverser, portant sur mon nouveau personnage un jugement ultime, lucide et objectif, je me classai délibérément parmi les entités « ni-acides-ni-alcalines », aussi neutres que les abeilles et les fourmis asexuées... aussi « anonymes-que-les-pyramides-d'Égypte » (V. Hugo).

N'empêche que j'aurais été curieuse de connaître l'avis de ceux qui, après La Bruyère, affirment qu' «Il faut juger les femmes depuis la chaussure jusqu'à la coiffure. » 


\section{Glose sur les « poches de sœurs »}

«Poches de sœurs! » légendaires fourre-tout! inséparables accessoires du costume féminin religieux d'une époque à jamais révolue! Salut à vous, ô siamoises vétustés !...

Épiloguons un bref instant sur vos profondeurs ténébreuses aux hétéroclites richesses. Pauvrettes !... à la mine infiniment triste de tout portefaix résigné, vous aviez un faux air de prison avec votre réjouissante couleur d'uniforme rayé des forçats...

Votre tissu, parlons-en ! du gros coutil épais, raide et dru ; quasiment aussi dru, aussi raide que des bâches de camions !... vos dimensions réglementaires ? longueur : seize pouces ; largeur : neuf pouces (peu importait la longueur et la largeur de la sœur !). C'était tant par tant ! « La loâ, c'est toujours la loâ. "

L'intérieur des poches était « renforcé » d'une large bande d'étoffe, très résistante, les coins, l'ouverture de ladite poche, également " doublés ", « fortifiés », « radoubés », par un non moins résistant galon pour défier l'usure.

C'est curieux !... comment, dès le noviciat, on nous faisait nager déjà par les six brasses dans le paradoxe! Ben oui ! D'une part, (côté révélé !) on nous enseignait l'éphémère, le fugace, le précaire des gens et des choses, pis, d'autre part, (côté praxis !) on nous harnachait pour durer !... J'en étais venue à croire, qu'en religion, l'important (oh non ! c'était pas la rose !) l'important, c'était de durer !!! Soit dit en passant, durer, ça use son homme en pas-pourrire $! . .$.

Dès que le port d'une montre s'avéra nécessaire, il fut statué de coudre à l'intérieur de la grosse poche, une pochette nouvelle pour éviter à la mignonne d'éventuelles égratignures. Telles quelles, et vides, nos deux pochetées latérales avaient déjà du poids... qu'allait devenir notre masse brute, une fois terminé l'arrimage des cargaisons futures ?...

Au tout début, mon attention avait été alertée par l'allure monotone des novices qui semblaient se dandiner comme... des mules au trot, cependant que très tôt $\mathrm{j}$ 'en vins par moi-même à expérimenter pourquoi nos jeunes échines tanguaient sans répit de bâbord à tribord... Simple affaire de lest, ma chère ! Centre de gravité plus ou moins stabilisé, rien que ça ! Mais inventorions plutôt le contenu des contenants en signalant que, dès le départ, nous devenions des spéléologues de métier : l'exploration à heures variables, dans nos gouffres latéraux, faisait remonter à la surface le matériau requis pour les besoins de la cause.

$1^{\circ}$ - Le livre des saintes Règles (sept pouces et demi par cinq et par trois quarts) recouvert d'une épaisse flanelle grise protectrice, poids total : une livre. 
$2^{\circ}$ - Une statuette de la Vierge, parfois en ivoirine ou en aluminium, mais le plus souvent en gros plomb ; proportions diverses ; art, le plus souvent douteux. Je ne me suis jamais opposée à la vénération d'une icône admirable. Il m'est arrivé toutefois d'éprouver une forte propension à épouser les querelles byzantines iconoclastes, lorsque, par exemple, je voyais des sœurs continuer de baiser et de s'attacher puérilement ou sénilement à des restes de statuettes devenues petites masses informes, sans relief ni vestiges de symbole. Ça me navrait et dégoûtait tout à la fois ce fétichisme aux simagrées de mauvais aloi.

$3^{\circ}$ - Un carnet de notes à prendre, sitôt la lecture spirituelle terminée ou selon l'envahissement des charismes de l'Esprit, bien qu'à l'époque, le terme " charisme " n'ait pas eu pignon sur rue.

$4^{\circ}$-Un second carnet : journal de bord, celui-là ; mise à jour de l'actif et du passif ; fameux code de l'examen de conscience. Cette cryptographie spirituelle m'a toujours déprimée (n'en déplaise à saint Ignace !). Étant perfectionniste à outrance et «Artaban » sur tous les bords, mes « victoires » et mes " progrès " ne figuraient que dans la colonne des... récessions. Je piquais alors des plongeons dans les marécages de l'hypocondrie...

$5^{\circ}$ - Un bout de crayon de mine : plus le bout était court, mieux c'était ! Humble témoignage d'une plus éclatante vertu de pauvreté... (ici, le paradoxe du « fini » à faire durer...)

$6^{\circ}$ - Une bouteille d'eau bénite (deux à quatre onces). C'est qu'on y croyait à l'efficacité des sacramentaux ! À preuve ! cette bonne sœur " doyenne » professant une telle confiance en la puissance de l'eau bénite, qu'elle croyait " exorciser les bébés protestants » en les aspergeant, entre parenthèses, comme si elle eut eu en main un vinaigrier ou une salière... Mais qui donc oserait reprocher à l'apôtre son zèle un tantinet intempestif ?... quant à argumenter sur l'orthodoxie de son opinion concernant l'exorcisme, je décline la responsabilité du « jet de la première pierre ", ça déborde le propos que je traite actuellement.

$7^{\circ}$ - Un trousseau de clés. À vrai dire, je n'ai jamais occupé une quelconque position-clé de ma vie. Mais, des tours de clés, j'en ai donné dans les placards, dans les lingeries, les pharmacies, les sacristies, les cagibis !... Quand on quittait son emploi, quand finissaient les heures « de garde » auprès des malades, tels les vaincus se soumettant aux vainqueurs, on présentait, on remettait, on rendait ses clés à la nouvelle vigie... Il n'y a que « prendre-laclé-des-champs » qui eût été impensable à l'époque, cependant qu'à la rigueur, une tentative de « prise-d'assaut-des-clés-du-Royaume » eût été facilement absolvable et qualifiée même de partance pour la gloire... 
Quoi qu'il en soit, le trousseau de clés venait ajouter son cliquetis sonore lorsqu'on le portait suspendu au bout d'une cordelette ou bien il augmentait le poids des poches quand on l'y enfouissait avec le reste du gréement.

$8^{\circ}$ - Une boîte en fer-blanc (trois pouces par deux) renfermant un trésor inestimable : un savon-pierre-ponce-très-spécial, à texture poreuse de couleur rosée, enveloppé d'une minuscule peau de chamois, le tout destiné au fourbissage de nos cuivres (crucifix et rosaire). Ça venait de France, ça coûtait des sous... c'était rare, il fallait ménager ça, mais que nos bijoux de cuivre étaient beaux !...

$9^{\circ}$ - Un gros couteau « de poche » à une ou deux lames, devant surtout servir comme couteau « de table ». Depuis 1703 , exactement, c'était la coutume à table de passer l'archaïque miche de pain sur laquelle, après avoir tracé la croix, chacune coupait la... tranche de son appétit. Le fameux couteau devait réintégrer la poche, sitôt le repas terminé, et ce, sans jamais connaître le moindre lavage. Le rituel en usage consistait à l'essuyer avec... de la mie de pain! J'en ai vu (des sœurs) pour qui cette mesure d'hygiène laissait des doutes, lécher la lame sans vergogne, voire même avec une onction et un recueillement dignes d'une meilleure cause... (mais coutume pour coutume ! au point où nous étions !!!)

$10^{\circ}$ - Une « ménagère » (onze par cinq et par un pouce, quatre onces), sorte de nécessaire de couture en tissu de flanelle blanche et grise compartimenté en replis tels les feuillets d'un bouquin. Chaque item avait sa case respective. Particularités : a) les quelques aiguillées de fil « à notre usage » étaient invariablement « roulées » sur des " carrés » de cartons découpés à la main ; b) un sachet de sable pour le dérouillement-dérouillage des aiguilles et des épingles d'acier. Hé oui ! tout nous rouillait sur le corps, « rapport » à la chaleur, à l'épaisseur et à la lourdeur du costume !...

$11^{\circ}$ - Un mouchoir de poche en grosse toile du pays, une demi-verge carrée (j'vous mens pas !) devant servir durant deux semaines environ, besoinpas-besoin ; rhume-pas-rhume ; sueurs-pas-sueurs. Laissez vagabonder votre imagination...

$12^{\circ}$-Une petite boîte ronde de pastilles Valda : genre-jujube-vert-foncéenrobé-de-sucre, au goût méli-mélo de créosote-eucalyptus-mentholathum, qu'importe ! ça faisait antiseptique et sécurisant contre d'éventuelles laryngites.

$13^{\circ}$ - Une queue-de-rat. (L'emploi ou le port de cet item fut épisodique et personnel à une époque de ma vie.) Pour être précise, je n'ai jamais vu la vraie queue d'un vrai rat (mammifère rongeur de la famille des muridés). J'ignore tout autant ce que c'est, et à quoi ça rime, cette queue-de-rat définie par le « Petit Robert » à la page 1435 , comme étant une « lime ronde et fine 
terminée en pointe ». Quand j'emploie le sobriquet, je désigne une interminable mèche enduite de suif et roulée sur elle-même en spirale. Toujours-z-estil, qu'un jour, je visitai une bonne " chère sœur » laquelle (en plus d'être musicienne, peintre, poète, collectionneuse de bric-à-brac dans une chambre meublée de bric et de broc), cette sœur, donc, était de surcroît un mécène débonnaire et désintéressé, la preuve ?...j'en hériterai une magnifique queuede-rat intacte ! Jusqu'à ce jour, cette précieuse denrée - apanage exclusif de nos vestales-sacristines - n'avait servi qu'à communiquer le feu aux cierges de l'autel. Allais-je en prostituer la noble fonction ?... ma gredine de conscience ne me posa aucun problème d'ordre moral et, le soir même, j'utilisais la mince flamme protégée au creux d'une boîte de conserves trouée, en guise de lampe de chevet, pour prolonger ma lecture au-delà de l'heure règlementaire ( 8 heures du soir), heure à laquelle il fallait éteindre l'électricité par souci d'économie (on était en temps de guerre).

Pour terminer cette interminable digression, je fais une mise au point en spécifiant que mes poches de sœurs ne pouvaient servir que de lieu de « transit » soir et matin aux fameuses queues-de-rat, « rapport» au... suif ! et, j'ajoute que ça faisait « début de colonie » en pas-pour-rire que de s'éclairer au sébum en... 1944 ! Comme j'ai hérité de mon père un goût pour le cocasse, le farfelu, le haut comique, ce qui est un peu fou a toujours trouvé grâce à mes yeux pour alléger le trop sérieux de la vie...

Le ciseau n'était pas dans la poche mais suspendu au bout d'un cordon de laine tressée, il pendait tout le long de la jambe droite (pas la gauche, même si vous étiez gauchère ! mais la droite) car, à senestre, était réservé le port de l'énorme chapelet aux mailles de cuivre agrémentées d'une non moins énorme médaille de la Vierge, également en cuivre. Les grains de ce rosaire avaient une particularité exotique : c'était de véritables noix d'Haïti ou d'Afrique. J'ai voulu, comme bien d'autres novices, juger par moi-même de l'identité de ces fruits : j'en ai cassé la coque déjà craquelée, j'en ai goûté l'amande, ça goûtait exactement la bonne petite noix normale quoiqu'un peu sèche qu'on aurait pu acheter chez « Laura Secord » quand Laura Secord avait des prix abordables pour des gens sous le secours direct... Autre détail : l'écale se polissait aux frottements répétés des doigts, aussi, les ave devenaient-ils d'un beau noir d'ébène lustrée. Toutefois, leur fragilité résistait mal aux chocs ou aux accrochages et, trop souvent, hélas ! nous avions des chapelets aux grains éventrés indignes des indulgences attachées à leur parfaite intégrité (?)... Combien attentionnées étions-nous à protéger ces lourdes fragilités de cinquante pouces de longueur (une dizaine mesurant déjà huit pouces !) le tout pesant une livre et quart, chaque grain ayant le poids et la grosseur d'une bille. Ouf $! . .$. 
Sans être le moins du monde tirée à quatre épingles, toute fille-de-lasagesse ne s'habillait pourtant qu'à grand renfort d'épingles. En effet, pas le moindre bouton, pas la moindre boutonnière ! Mentionnons en passant l'énorme, l'unique, la monumentale agrafe en cuivre jaune ou rouge qui bouclait la ceinture de notre jupe de bure aux dix-huit plis à la taille... À part ce renfort, point n'était besoin de tirer jamais ses épingles du jeu... en perdre une équivalait quasiment friser la catastrophe, quand je vous confierai qu'il fallait exactement neuf fortes épingles d'acier d'un pouce et quart de longueur pour tenir ensemble le plus gros du « harnais » officiel... (costume inchangé depuis l'an de grâce 1703, modifié légèrement en l'année 1966). Notre coiffe, à elle seule, fragile édifice empesé, compliqué à souhait, était maintenue en équilibre grâce à un savant épinglage de treize épingles moyennes, toutes et chacune en position stratégique pour stabiliser l'échafaudage instable. Ajouter ou enlever une seule épingle de l'habit monastique constituait un réel accroc aux immuables coutumes ancestrales.

Donc, à côté du pendentif-ciseau, s'ajoutait le pendentif pelote-à-épingles. Ce coussinet de sable (deux pouces par deux pouces) était décoré d'idéogrammes ésotériques : JHS, JMJ, DS, MJ. La croix grecque ou celle de saint André y figurait avec la croix latine. Chaque sœur s'ingéniait à piquer et à disposer ses têtes d'épingles selon ses goûts et ses dévotions... Trois catégories d'épingles avaient cours chez nous : les épingles d'acier, les épingles ordinaires et les «petites-sagesses ». Ces trois catégories portaient des dénominations diverses : épingles de cou, épingles de mouchoir, épingles de cape, épingles de coiffe, selon leur fonction.

Les épingles d'acier constituaient le plus précieux de notre patrimoine, celui sans lequel on ne pouvait « tenir »... I mean « tenir-ensemble-commele-Canada-ou-comme-la-peau-des-vaches » (Frère Untel)... En effet, notre habit « tenait ensemble » en autant qu'à Noël on recevait sa douzaine d'épingles d'acier de saint Nicolas. Ça venait de France et c'était un butin archiprécieux, voire indispensable.

Les épingles, format ordinaire, étaient sans histoire. Quant aux « petitessagesses ", ces minuscules tiges de métal pointues (un demi-pouce de longueur) réservées à l'unique usage de l'épinglé du col du mouchoir de cou, je me suis maintes fois demandé d'où leur venait cette appellation. Comme j'excelle dans le calcul et le raisonnement dont « la marque propre est d'être une opération médiate qui a pour terme une conclusion, " (vous me suivez ?...) j'en suis arrivée à cette déduction lumineuse, à savoir :

Prémisse $n^{\circ} 1-$ «Petites-sagesses » on les appelait ainsi depuis le 2 février 1703 ;

Prémisse $n^{\circ} 2$ - Or... étant admise à l'immutabilité des us et coutumes, ça ne pouvait pas « mouver $» . .$. 
Donc, eh bien ! conclusion purement formelle, étrangère au réel comme tout bon syllogisme !...

Mais je crois avoir suffisamment monté mon sujet... en épingles, pour passer au dédouanage du « fret » si, toutefois, il en reste à fond de cale.

Toujours dans le système des penderies « gauche-droite-au-pas-camarades-au-pas ! " je dois mentionner la corde bénite (deux à trois verges de longueur) nouée à la taille par un nœud coulant et agrémentée de trois autres gros nœuds à espaces réguliers en rappel de nos trois vœux. Quand, par maladresse ou précipitation, on se heurtait à une surface dure et qu'on « frappait.. un nœud » il va sans dire qu'on récoltait... un bleu !

Il y avait enfin la chaînette du Saint Esclavage prônée par notre fondateur comme symbolisme de service et d'attachement envers la Vierge Marie. Cette chaînette de longueur variable (douze à seize pouces) était en métal léger, à grosses mailles lâches, elle aussi pendait librement, peu importait le côté.

Voilà !... C'est ainsi que nous allions, sellées comme l'ânesse de Balaam, nos deux poches (reliées entre elles par un extra-fort en coton gris-fer) nous battant les flancs à longueur de journée ! Chacun de nos pas imprimait à nos multiples pendentifs le mouvement de pendule : " rappel constant de la fameuse découverte de Galilée concernant la loi des oscillations isochroniques pendulaires $\gg .$. cependant que, plus moderne dans la description de mes sensations, j'avais l'exaltante impression de participer incessamment à un marathon de ping-pong endiablé !

Le lest règlementaire de nos poches de sœurs, pour impressionnant qu'il fut, n'a jamais entravé l'entrée libre du " marché noir »... Tout ce qui se compte, s'entasse, se ramasse, s'empile, se plie, s'entortille, s'enroule et s'emmêle, trouvait gîte dans ces fameux marchés ambulants.

Le costume, dans son entier, pesait vingt-cinq livres. Inutile d'insister ! Tout l'charroi donnait grand air à chacune de nos démarches. Indéniablement, nous étions ce que l'on peut appeler : des femmes de poids !

C'est ainsi, qu'au lyrique «Amor pondus » de saint Augustin, j'ai toujours rabattu fièrement le dyptique de mon «Vestimentum pondus ». On a le style lapidaire qu'on peut !

Vieilles « poches-de-sœurs », vieilles sœurs... pochetées (!) votre profil se dilue en d'évanescentes nues...

frusques d'une hégire close, reposez dans vos replis, là, où gisent tant de choses au souterrain de l'oubli... 